\title{
Überlegungen zur Digitalen Archäologie und zu ihrem Verhältnis zu den Digitalen Geisteswissen- schaften
}

\author{
Dominik Hagmann
}

\author{
Universität Wien, Institut für Klassische Archäologie \\ dominik.hagmann@univie.ac.at \\ https://homepage.univie.ac.at/dominik.hagmann
}

Keywords: Digital Archaeology, Theory, Taxonomy, Academic Fields

\begin{abstract}
What does the term 'Digital Archeology' exactly mean and how does it relate to the Digital Humanities? The paper wants to take up as well as critically address just that question and gives a short outlook on further considerations.
\end{abstract}

\section{Fragestellung}

Was kann der Begriff „Digitale Archäologie“ (DA) genau bedeuten und in welcher Beziehung steht er zu den "Digitalen Geisteswissenschaften" (DG)? Der nachfolgende Beitrag will diese Frage aufgreifen und kritisch behandeln sowie einen kurzen Ausblick auf weitere Überlegungen geben.

\section{Versuch einer Charakterisierung der „Digitalen Archäologie“}

„Archäologie“ widmet sich der Erforschung der materiellen Kultur vergangener Zeiten ${ }^{1}$, unter der Verwendung verschiedenster Methoden und Theorien. ${ }^{2}$ Bei „Archäologie“ handelt es sich jedoch nicht um ein einziges Fach - vielmehr ist eine eindeutige Charakterisierung kaum möglich, da eine Vielfalt an speziellen (interdisziplinären) Ausrichtungen ${ }^{3}$ innerhalb des Faches auszumachen ist. So gliedert sich „Archäologie" in teilweise vollkommen unabhängige Teilfächer, die ihrerseits von verschiedenen Forschungstraditionen geprägt sind. Diese Vielfalt ist dabei inklusiv und die Grenzen sind nicht eindeutig: Kulturwissenschaftliche Analysen sind ebenso üblich wie bioarchäologische Studien von Tierresten zur Rekonstruktion des Ernährungsverhaltens.

Die beschriebene Vielfalt kann wohl als einer der Gründe angesehen werden, dass es bereits unmittelbar nach dem Aufkommen computergestützter Verfahren zu ersten Gehversuchen im Bereich datengetriebener archäologischer Studien kam. ${ }^{4}$ Seit der Verfügbarkeit von digitalen Informations-

1 Vgl. zu einer Definition etwa Mikkel Bilte / Tim Flohr Sørensen, Material Culture, in: Sandra L. López Varela, Hg., The Encyclopedia of Archaeological Sciences, Malden 2018, 1-5.

2 Jane Balme, Artifacts, Overview, in: Deborah Marie Pearsall, Hg., Encyclopedia of Archaeology, San Diego 2008, 508517, hier 509; Manfred K. H. EgGert, Prähistorische Archäologie. Konzepte und Methoden, 4. Auflage, Tübingen 2012; Tonio HölsCher, Klassische Archäologie. Grundwissen, 4. Auflage, Darmstadt 2015; Vgl. hierzu etwa Colin RenfREw / Paul G. BAHN, Archaeology. Theories, Methods and Practice, 7. Auflage, London 2016.

3 Im Folgenden als Teildisziplinen, -fächer und dergleichen bezeichnet.

4 Ethan Watrall, Archaeology, the Digital Humanities, and the "Big Tent", in: Matthew K. Gold / Lauren F. Klein, Hg., Debates in the Digital Humanities 2016, Minneapolis 2016, 345-358, hier 346-349. 
und Kommunikationstechnologien (IKT) werden diese ebenso für archäologische Fragestellungen herangezogen, anfangs besonders um mit modernen Methoden effektiv große Datenmengen zu erzeugen und zu verarbeiten. ${ }^{5}$ Dieser Umstand wird von P. T. Daly und T. L. Evans in ihrem Standardwerk zur DA aus dem Jahr 2006 eindeutig beschrieben: „Digital Archaeology explores the basic relationships that archaeologists have with Information and Communication Technology (ICT) and digital technology to assess the impact that such innovations have had on the very basic ways that archaeology is performed and considered." Nach über 50 Jahren an archäologischer Arbeit in diesem Gebiet kann diese Definition einer „Fusion“ von Informatik und Archäologie(n) um weitere Merkmale ergänzt werden, wie etwa um die Rolle der digitalen Public Archaeology als inklusives Element zwischen Wissenschaft und Gesellschaft im Bereich der DA. ${ }^{7}$ Auch muss die Ubiquität digitaler Methoden ${ }^{8}$ berücksichtigt werden oftmals wird deren Einsatz bereits als gegeben vorausgesetzt und nicht (mehr) als explizites Merkmal der DA gesehen. ${ }^{9}$ Demnach obliegt es der DA, nicht "nur“ die bloße Verwendung von digitalen Tools zu behandel $\mathrm{n}^{10}$, sondern vielmehr auch die theoretische Auseinandersetzung mit der Materie zu forcieren: „All archaeologies can be digital, but not all archaeologies are Digital Archaeology.“11

Der Umstand, verschiedene IKT zur Erforschung der materiellen Kultur praktisch einzusetzen und auch theoretisch zu behandeln, wird mit verschiedenen Termini wie „DA“12, „Virtuelle Archäologie“13 oder "Cyber-Archäologie"14 beschrieben. Diese verfügen jeweils über konkrete Ausprägungen und gegebenenfalls auch bestimmte Abhängigkeiten zueinander. ${ }^{15}$ Nicht zuletzt kann zudem festgestellt werden, dass einzelne Begriffe zu gewissen Zeitpunkten als trending terms besonders in Mode sind. ${ }^{16}$

Doch was meint der Begriff „DA“, der hier als Platzhalter für die diversen Termini Verwendung findet, eigentlich? Bei genauerer Betrachtung stellt sich schnell die Frage, ob sich kongruent zur jeweiligen Fachrichtung (z. B. Prähistorische Archäologie, Klassische Archäologie etc.) mit „DA“ jeweils eine eigene digitale Teildisziplin etabliert. Muss in weiterer Folge also im Grunde von „Digitaler Prähistorischer“ und „Digitaler Klassischer Archäologie“ gesprochen werden und handelt es sich dabei um Schwesternwissenschaften? Sollen diese Teilfächer dann in einem weiteren Schritt unabhängig voneinander institutionalisiert oder in einer selbstständigen Disziplin „DA“ subsummiert werden? Übernimmt diese DA (womöglich auch als Institution) danach alle wissenschaftlichen und lehrbezogenen Zuständigkeiten?

5 Shawn Graham u. a., The Open Digital Archaeology Textbook 2019-ongoing, online unter: https://o-date.github.io/draft/ book/so-what-is-digital-archaeology.html\#a-distant-view (12.03.2019); Patrick T. DALY / Thomas L. Evans, Introduction. Archaeological Theory and Digital Pasts, in: Thomas L. Evans / Patrick T. Daly, Hg., Digital Archaeology: Bridging Method and Theory, London u.a. 2006, 2-7.

6 DALY / EVANS, Introduction, 2.

7 Graham, Open; Dominik Hagmann, Reflections on the Use of Social Networking Sites as an Interactive Tool for Data Dissemination in Digital Archaeology, in: Interdisciplinaria Archaeologica 9/1 (2018), 7-20; Lorna-Jane RICHARDSON / Jaime Almansa-SÁnchez, Do You Even Know What Public Archaeology Is? Trends, Theory, Practice, Ethics, in: World Archaeology 47/2 (2015), 194-211; Lorna-Jane RICHARDSON, Ethical Challenges in Digital Public Archaeology, in: Journal of Computer Applications in Archaeology 1/1 (2018), 64-73.

8 William R. CARAHER, Continuity, Practice, and Theory in Digital Archaeology, online unter: https://mediterraneanworld.wordpress.com/2018/12/17/continuity-practice-and-theory-in-digital-archaeology/ (24.01.2019).

9 Andre Costopoulos, Digital Archeology Is Here (and Has Been for a While), in: Frontiers in Digital Humanities 3 (2016).

10 Graham, Open.

11 Ebd.

12 Ezra B. W. ZuBrow, Digital Archaeology. A Historical Context, in: Thomas L. Evans / Patrick T. Daly, Hg., Digital Archaeology: Bridging Method and Theory, London u.a. 2006, 8-26.

13 Paul ReIlly, Towards a Virtual Archaeology, in: Sebastian Rahtz / Kris Lockyear, Hg., CAA90: Computer Applications and Quantitative Methods in Archaeology 1990, Oxford 1991, 132-139.

14 Thomas Evan Levy u. a., Cyber-Archaeology in the Holy Land. The Future of the Past, San Diego 2012.

15 Ebd., 4.

16 Jeremy Huggett, Core or Periphery? Digital Humanities from an Archaeological Perspective, in: Historical Social Research: Historische Sozialforschung 37/3 (2012), 86-105, hier 90. 
Oder handelt es sich bei DA eher um die Bezeichnung eines „Phänomens“, das zwar die Anwendung von IKT in der Archäologie in Theorie und Praxis beschreibt, aber jedem einzelnen Teilfach im Rahmen der „regulären“ archäologischen Arbeit ohnehin ständig innewohnend ist? Könnte dies in weitere Folge also heißen, dass es sich bei DA zwar um die Auseinandersetzung mit IKT innerhalb eines archäologischen Fachbereichs handelt, diese sich jedoch nicht zu einem dezidierten Fach, sondern zu einer Metadisziplin ausformt? ${ }^{17}$

\section{Das Verhältnis von DA und DG}

Die fachliche Variabilität der Archäologie ist das Hauptproblem der taxonomischen Verortung der Beziehung der DA und DG zueinander, denn die archäologische Disziplinenlandschaft wird keineswegs ausschließlich den Geisteswissenschaften zugeordnet - besonders im anglo-amerikanischen Raum wird Archäologie oftmals als Sozialwissenschaft betrachtet. ${ }^{18}$ In Mitteleuropa werden hingegen in taxonomischer Hinsicht die „Archäologien“ meist den Geisteswissenschaften zugeteilt. ${ }^{19}$

Betrachtet man Archäologie nun als Geisteswissenschaft, kann rein taxonomisch auch eine Verbindung zwischen den jeweiligen digitalen Bereichen hergestellt werden. In Anbetracht der zuvor angestellten Überlegungen gilt es hierfür unmittelbar in einem weiteren Schritt zu klären, was genau diese digitalen Bereiche in beiden Fällen darstellen sollen: spezialisiertes Arbeiten innerhalb eines Teilfachs oder selbstständige Fächer? Handelt es sich dann bei DA um eine Subdisziplin der DG ${ }^{20}$ Oder bestehen DA und DG dennoch ohne Verbindung nebeneinander? Definiert man Archäologie hingegen als sozialwissenschaftlich orientiertes Fach, stellt sich die Frage, ob sich dementsprechend auch die Beziehung der DA zur DG ändert. Sind in diesem Fall DG und DA zwar strukturell gesehen vergleichbar, aber nicht voneinander abhängig, wie es besonders von sozialwissenschaftlich orientierten Archäolog* innen gesehen wird ${ }^{21}$ ?

\section{Offene Fragen und Ausblick}

Was ist also DA? Diese Frage kann hier vorerst nicht beantwortet werden. Die DA fungiert als Schnittstelle für alle Aspekte der Digitalisierung zwischen diversen archäologischen Teilfächern und kann als Spezialisierung gelten. ${ }^{22}$ DA bietet die Möglichkeit, digitale Methoden auszuwählen, fachgerecht umzusetzen und dies praktisch sowie theoretisch zu evaluieren. Weniger scheint man DA hingegen als eine Fachdisziplin umreißen, einem Fach zuordnen oder einer Forschungstradition zuweisen zu können. Zu beachten ist, dass die Entwicklung der DA trotz allem nach wie vor erst am Anfang steht.

Nur ihre langfristige Beobachtung kann womöglich Aufschluss über die offenen Fragen geben. ${ }^{23}$ Die Frage nach dem genauen Verhältnis von DA und DG konnte hier ebenso vorerst nicht eindeutig

17 Hagmann, Reflections, $9 f$.

18 Watrall, Archaeology, $349 f$.

19 Statistik Austria, Österreichische Systematik der Wissenschaftszweige (ÖFOS) 2012, online unter: https://www.data.gv.at/ katalog/dataset/stat_ofos-2012 (24.04.2019).

20 Vgl. hierzu das 3-Sphären-Modell zur Kartierung der Digital Humanities in Patrick SAHLE, Digital Humanities? Gibt's doch gar nicht!, in: Sonderband der Zeitschrift für digitale Geisteswissenschaften 1 (2015), hier 7.

21 WatraLL, Archaeology.

22 Katy MEYERS, Defining Digital Archaeology, online unter: http://chi.anthropology.msu.edu/2011/10/defining-digital-archaeology/ (12.03.2019).

23 Erin Walcek Averett / Jody Michael Gordon / Derek B. Counts, Mobile Computing in Archaeology. Exploring and Interpreting Current Practices, in: Erin Walcek Averett u. a., Hg., Mobilizing the Past for a Digital Future: The Potential of Digital Archaeology, Grand Forks 2016, 1-30, hier 9. 
beantwortet werden. Es muss weiterführend gefragt werden, ob eine präzise taxonomische Verortung tatsächlich der richtige Ansatz ist, denn je nach Forschungstradition und genauer fachlicher Ausrichtung kommt es zur Anwendung unterschiedlicher Systematiken und damit zu unterschiedlichen Ergebnissen - eine pauschale Antwort ist jedenfalls nicht möglich.

Unabhängig von der angerissenen Problematik besitzen die DG jedenfalls das Potential als „Brücke“ zwischen den digital betriebenen Archäologien und Geisteswissenschaften zu dienen. ${ }^{24}$ Bestes Beispiel für eine praktikable Umsetzung dieses Gedankens sind Tagungen wie die DHA selbst, die eine Plattform für eine derartige Vernetzung darstellen und diese ebenso fördern.

\section{Literaturverzeichnis}

Erin Walcek Averett / Jody Michael Gordon / Derek B. Counts, Mobile Computing in Archaeology. Exploring and Interpreting Current Practices, in: Erin Walcek Averett u. a., Hg., Mobilizing the Past for a Digital Future: The Potential of Digital Archaeology, Grand Forks 2016, 1-30.

Jane BALme, Artifacts, Overview, in: Deborah Marie Pearsall, Hg., Encyclopedia of Archaeology, San Diego 2008, 508-517.

Mikkel BıLLE / Tim Flohr SøRensen, Material Culture, in: Sandra L. López Varela, Hg., The Encyclopedia of Archaeological Sciences, Malden 2018, 1-5.

William R. CARAHER, Continuity, Practice, and Theory in Digital Archaeology, online unter: https://mediterraneanworld.wordpress.com/2018/12/17/continuity-practice-and-theory-in-digital-archaeology/ (24.01.2019).

Andre Costopoulos, Digital Archeology Is Here (and Has Been for a While), in: Frontiers in Digital Humanities 3 (2016), DOI: 10.3389/fdigh.2016.00004.

Patrick T. Daly / Thomas L. Evans, Introduction. Archaeological Theory and Digital Pasts, in: Thomas L. Evans / Patrick T. Daly, Hg., Digital Archaeology: Bridging Method and Theory, London u.a. 2006, 2-7.

Manfred K. H. Eggert, Prähistorische Archäologie. Konzepte und Methoden, 4. Auflage, Tübingen 2012.

Shawn Graham u. a., The Open Digital Archaeology Textbook 2019-ongoing, online unter: https://o-date.github. io/draft/book/so-what-is-digital-archaeology.html\#a-distant-view (12.03.2019).

Dominik Hagmann, Reflections on the Use of Social Networking Sites as an Interactive Tool for Data Dissemination in Digital Archaeology, in: Interdisciplinaria Archaeologica 9/1 (2018), 7-20.

Tonio HöLSCHER, Klassische Archäologie. Grundwissen, 4. Auflage, Darmstadt 2015.

Jeremy Huggett, Core or Periphery? Digital Humanities from an Archaeological Perspective, in: Historical Social Research: Historische Sozialforschung 37/3 (2012), 86-105.

Thomas Evan Levy u. a., Cyber-Archaeology in the Holy Land. The Future of the Past, San Diego 2012.

Katy MEYERS, Defining Digital Archaeology, online unter: http://chi.anthropology.msu.edu/2011/10/defining-digital-archaeology/ (12.03.2019).

Paul ReILly, Towards a Virtual Archaeology, in: Sebastian Rahtz / Kris Lockyear, Hg., CAA90: Computer Applications and Quantitative Methods in Archaeology 1990, Oxford 1991, 132-139.

Colin Renfrew / Paul G. Bahn, Archaeology. Theories, Methods and Practice, 7. Auflage, London 2016.

Lorna-Jane RichARDSON, Ethical Challenges in Digital Public Archaeology, in: Journal of Computer Applications in Archaeology 1/1 (2018), 64-73.

Lorna-Jane Richardson / Jaime Almansa-Sánchez, Do You Even Know What Public Archaeology Is? Trends, Theory, Practice, Ethics, in: World Archaeology 47/2 (2015), 194-211.

24 Huggett, Core, 101. 
Patrick SAHLE, Digital Humanities? Gibt's doch gar nicht!, in: Sonderband der Zeitschrift für digitale Geisteswissenschaften 1 (2015), DOI: 10.17175/sb001_004.

Statistik Austria, Österreichische Systematik der Wissenschaftszweige (ÖFOS) 2012, online unter: https://www. data.gv.at/katalog/dataset/stat_ofos-2012 (24.04.2019).

Ethan Watrall, Archaeology, the Digital Humanities, and the "Big Tent", in: Matthew K. Gold / Lauren F. Klein, Hg., Debates in the Digital Humanities 2016, Minneapolis 2016, 345-358.

Ezra B. W. ZuBrow, Digital Archaeology. A Historical Context, in: Thomas L. Evans / Patrick T. Daly, Hg., Digital Archaeology: Bridging Method and Theory, London u.a. 2006, 8-26. 